\title{
Treatment of medulloblastoma in children and adolescents
}

\section{TRATAMENTO DO MEDULOBLASTOMA EM CRIANC AS E ADOLESCENTES}

Nelci Zanon Collange ${ }^{1}$, Silvana de Azevedo Brito ${ }^{1}$, Ricardo Rezende Campos ${ }^{1}$, Egmond Alves Silva Santos ${ }^{1}$,

Ricardo Vieira Botelho ${ }^{1}$

1. Medulloblastoma in children should consider:

a. Medulloblastoma can be cured if diagnosed early.

b. Prognosis for medulloblastoma depends on the severity of hydrocephalus, size of the tumor, and whether there is brain-stem invasion.

c. The age of onset of medulloblastoma has no relation to the prognosis.

d. Medulloblastoma should be considered a serious illness in children.

2. Surgical resection:

a. Does not change the prognosis.

b. Can be performed for biopsy alone, since treatment is chemotherapy.

c. Complete resection should be attempted if possible as it improves the prognosis.

d. Resection of brain-stem tumor should be avoided.

3. On the effect of tumor resection for the resolution of hydrocephalus:

a. All patients require ventricular shunt.

b. All patients require external ventricular shunt during resection.

c. Most patients achieve resolution of hydrocephalus after tumor removal.

d. Hydrocephalus resolves after chemotherapy.

4. Radiotherapy associated with the treatment of medulloblastoma:

a. Should not be performed; only chemotherapy is indicated.

b. Should be avoided in children under 3 years old.

c. Should be performed on the skull and spine.

d. Should be performed only in the skull.

e. Two of the above conditions are true.

5. On the disease-free interval after treatment:

a. The approximate disease-free survival at 5 years is $50 \%$.

b. Tumor recurrence, if any, occurs after 15 years of treatment. c. The presence of brain-stem invasion is related to the prognosis.

d. Patients with hydrocephalus have a shorter tumorfree period following surgery.

\section{ANSWERS TO CLINICAL SCENARIO: UPDATE ON ZIKA VIRUS INFECTION IN PREGNANCY [PUBLISHED IN RAMB 2016; 62(2)]}

1. Which factors may be related to the substantial increase in the number of cases of microcephaly in Brazil?

Increased active search for this congenital malformation, and change in diagnostic criteria. (Alternatives A and $\mathbf{C}$ )

2. What is the clinical and laboratory method indicated (sensitivity and specificity) to confirm Zika virus infection within a week of the onset of symptoms?

Real-time polymerase chain reaction (RT-PCR). (Alternative $\mathbf{D})$

3. Regarding symptoms in pregnant women with suspected Zika virus infection, we know that: Most are asymptomatic but they can manifest fever, rash and fatigue. (Alternatives $\mathbf{A}$ and $\mathbf{C}$ )

4. What treatments are recommended for people infected with Zika virus?

Hydration, rest and non-salicylic analgesic drugs. (Alternative $\mathbf{C}$ )

5. Regarding pregnant women diagnosed with Zika virus infection, we know that:

Fetal ultrasounds must be done in series every 3 to 4 weeks, and the newborn should receive complete physical examination with ophthalmologic assessment. (Alternative B) 